hep-th/9709006

\title{
Membrane, Four-Brane and Dual Coordinates in the M(atrix) Theory Compactified on Tori
}

\author{
Shijong Ryang \\ Department of Physics \\ Kyoto Prefectural University of Medicine \\ Taishogun, Kyoto 603 Japan
}

\begin{abstract}
In the $\mathrm{M}$ (atrix) theory by making the expansions of the matrices around the membrane and four-brane solutions we derive the three- and five-dimensional gauge theories on the dual tori. The explicit forms of solutions yield the dual coordinates and each expansion is related to a toroidal compactification of the M(atrix) theory. From the derived Lorentz and gauge invariant actions the gauge coupling constants are shown to be characterized by the volume of the dual tori.
\end{abstract}

August, 1997 
The M(atrix) theory has been proposed as a nonperturbative microscopic description of M-theory [1]. The dynamics of M-theory in the infinite momentum frame is described by the large N 10-dimensional U(N) Super Yang Mills theory (SYM) reduced to $0+1$ dimensions whose Hamiltonian consists of D0-branes and their interactions. Its operatorlike classical solutions are identified with $\mathrm{D} p$-branes of the type IIA superstring theory. The long-distance interactions of some $p$-branes have been shown to be in agreement with supergravity predictions [2, 国, 田].

The M(atrix) theory description of toroidal compactification has been given by Ganor, Ramgoolam and Taylor [5, 6], where the $\mathrm{M}\left(\right.$ atrix) theory on $T^{3}$ is T-duality equivalent to the U(N) SYM theory on the dual torus $\hat{T}^{3}$ and T-duality is realized by S-duality in the 4-dimensional SYM theory. On the other hand starting from the Lagrangian of the $\mathrm{U}(\mathrm{N})$ SYM theory in $0+1$ dimensions and analyzing the small fluctuation around the general classical $p$-brane solution Banks, Seiberg and Shenker have derived the U(N) SYM theory in $p+1$ dimensions, which describes D $p$-brane in the IIA string theory [0]. In this derivation the notions of T-duality as well as toroidal compactification are absent. We will fill up the gap between the two approaches. By using the explicit infinite membrane and four-brane solutions and extracting dual variables from them we will derive the gauge invariant actions on the dual spacetimes.

We start with the Lagrangian of the $\mathrm{M}($ atrix) theory described by the $\mathrm{U}(\mathrm{N})$ supersymmetric quantum mechanics $S Y M_{0+1}$ [1]. The bosonic part of it is given by

$$
L=\frac{1}{2 g_{s}} \operatorname{Tr}\left(\frac{1}{\alpha^{\prime 2}} D_{0} X^{i} D_{0} X^{i}+\frac{1}{2 \alpha^{\prime 4}}\left[X^{i}, X^{j}\right]^{2}\right),
$$

where $D_{0}=\partial_{t_{0}}-i\left[A_{0}, \cdot\right]$ and $i=1,2, \cdots, 9$ [8]. The string coupling constant $g_{s}$ and $X^{i}$ have mass dimensions 3 and -1 respectively. Through the rescalings such as $Y=$ $X / g^{1 / 3}, t=t_{0} / g^{1 / 3}$ and $g_{s}=g\left(\alpha^{\prime}\right)^{-3 / 2}$ this Lagrangian can be rewritten as

$$
L=\operatorname{Tr}\left(\frac{1}{2 R} D_{t} Y^{i} D_{t} Y^{i}+\frac{R}{4 \alpha^{\prime 3}}\left[Y^{i}, Y^{j}\right]^{2}\right),
$$

where $R=g^{2 / 3} \sqrt{\alpha^{\prime}}$.

We take an infinite membrane background stretched out in the 1,2 planes to be

$$
U_{1}=-\sqrt{N} R_{1} p, \quad U_{2}=\sqrt{N} R_{2} q
$$

with all others $U_{I}=0, I=3, \cdots, 9$ where $R_{r}, r=1,2$ are the lengths of the corresponding directions and $[q, p]=2 \pi i / N$ [四, 2]. The minus sign of $U_{1}$ is suggested by the construction of D-string action [9] from the D-instanton matrix model [10]. Following the general framework of Ref. [7], we expand the matrix variables around the explicit membrane background

$$
Y^{1}=U_{1}+2 \pi \alpha^{\prime} A_{1}, \quad Y^{2}=U_{2}+2 \pi \alpha^{\prime} A_{2}, \quad Y^{I}=Z_{I} .
$$


Here we define dual matrices $x_{1}, x_{2}$ as

$$
x_{1}=\frac{\alpha^{\prime} \sqrt{N}}{R_{1}} q, \quad x_{2}=\frac{\alpha^{\prime} \sqrt{N}}{R_{2}} p
$$

and calculate commutation relations to have

$$
\begin{gathered}
{\left[Y^{1}, Y^{2}\right]=2 \pi i R_{1} R_{2}+\left(2 \pi \alpha^{\prime}\right)^{2} i F_{12},} \\
D_{t} Y^{r}=2 \pi \alpha^{\prime} F_{t r}, r=1,2, \\
{\left[Y^{r}, Y^{I}\right]=2 \pi \alpha^{\prime} i D_{r} Z_{I},}
\end{gathered}
$$

where $F_{12}=\partial_{x_{1}} A_{2}-\partial_{x_{2}} A_{1}-i\left[A_{1}, A_{2}\right]$. From (14) and (61) the Lagrangian (2) can be expanded as

$$
\begin{aligned}
L= & \operatorname{Tr}\left(\frac{\left(2 \pi \alpha^{\prime}\right)^{2}}{2 R} F_{t r}^{2}-\frac{(2 \pi)^{4} R \alpha^{\prime}}{2} F_{12}^{2}-\frac{2 \pi^{2} R}{\alpha^{\prime 3}}\left(R_{1} R_{2}\right)^{2}\right. \\
& \left.+\frac{1}{2 R}\left(D_{t} Z_{I}\right)^{2}-\frac{2 \pi^{2} R}{\alpha^{\prime}}\left(D_{r} Z_{I}\right)^{2}+\frac{R}{4 \alpha^{\prime 3}}\left[Z_{I}, Z_{J}\right]^{2}\right) .
\end{aligned}
$$

In this expansion the equations of motion for the static configuration are taken into account by dropping linear terms in the fluctuations.

In the $A_{t}=0$ gauge a Hamiltonian is provided by

$$
\begin{aligned}
H= & R \operatorname{Tr}\left(\frac{1}{2\left(2 \pi \alpha^{\prime}\right)^{2}} E_{r}^{2}+\frac{(2 \pi)^{4} \alpha^{\prime}}{2} F_{12}^{2}+\frac{1}{2} \Pi_{I}^{2}\right. \\
& \left.+\frac{2 \pi^{2}}{\alpha^{\prime}}\left(D_{r} Z_{I}\right)^{2}+\frac{2 \pi^{2}}{\alpha^{\prime 3}}\left(R_{1} R_{2}\right)^{2}-\frac{1}{4 \alpha^{\prime 3}}\left[Z_{I}, Z_{J}\right]^{2}\right) .
\end{aligned}
$$

We will consider the membranes of toroidal topologies in the large $\mathrm{N}$ limit. Using a pair of unitary operators $U, V$ satisfying $U V=\omega V U, U^{N}=V^{N}=1$ with $\omega=\exp (2 \pi i / N)$ we have a set of matrices $J_{\boldsymbol{m}}=\omega^{-m_{1} m_{2} / 2} U^{m_{1}} V^{m_{2}}$ for $\boldsymbol{m}=\left(m_{1}, m_{2}\right)$ and $0 \leq m_{1}, m_{2} \leq N-1$, which include $J_{00}$ and are a linearly independent, complete set of basis for $\mathrm{U}(\mathrm{N})$ matrices. The fluctuations around the background and their conjugate momenta are expanded in the form

$$
\begin{array}{cc}
A_{r}=\frac{1}{2 \pi \sqrt{\alpha^{\prime}}} \sum_{\boldsymbol{m}} A_{\boldsymbol{m}}^{r} J_{\boldsymbol{m}}, & E_{r}=2 \pi \sqrt{\alpha^{\prime}} \sum_{\boldsymbol{m}} E_{\boldsymbol{m}}^{r} J_{\boldsymbol{m}}, \\
Z_{I}=\sqrt{\alpha^{\prime}} \sum_{\boldsymbol{m}} Z_{\boldsymbol{m}}^{I} J_{\boldsymbol{m}}, & \Pi_{I}=\frac{1}{\sqrt{\alpha^{\prime}}} \sum_{\boldsymbol{m}} \Pi_{\boldsymbol{m}}^{I} J_{\boldsymbol{m}} .
\end{array}
$$

The matrices $J_{\boldsymbol{m}}$ satisfy an orthonormal relation

$$
\operatorname{Tr} J_{\boldsymbol{m}} J_{\boldsymbol{n}}=N \delta_{m_{1}+n_{1}, 0} \delta_{m_{2}+n_{2}, 0},
$$


where $\delta$ is a mod.N delta function. Since $U$ and $V$ are realized to be exponentials of the canonical variables $p, q$ as $U=e^{i p}, V=e^{i q}$ the expressions (9) are understood as the mode expansions in the quantum phase space $(p, q)$. Using $p$ and $q$ leads to the alternative expression $J_{\boldsymbol{m}}(p, q)=\exp \left[i\left(m_{1} p+m_{2} q\right)\right]$. By plugging the mode expansions (9) into the Hamiltonian (8) and taking the trace by means of (10) we obtain the decomposition

$$
\begin{aligned}
H= & R\left(H_{0}+H_{1}+H_{2}\right), \\
H_{0}= & N\left(\frac{1}{2 \alpha^{\prime}} \sum_{\boldsymbol{m}}\left(\Pi_{-}^{I} \boldsymbol{m} \Pi_{\boldsymbol{m}}^{I}+E_{-\boldsymbol{m}}^{r} E_{\boldsymbol{m}}^{r}\right)+\frac{2}{\alpha^{\prime 3}}\left(\pi R_{1} R_{2}\right)^{2}\right) \\
& +\frac{2 \pi^{2}}{\alpha^{\prime 2}} \sum_{\boldsymbol{m}}\left(\left(R_{1} m_{2} A_{-\boldsymbol{m}}^{2}-R_{2} m_{1} A_{-\boldsymbol{m}}^{1}\right)\left(R_{1} m_{2} A_{\boldsymbol{m}}^{2}-R_{2} m_{1} A_{\boldsymbol{m}}^{1}\right)+\omega_{\boldsymbol{m}}^{2} Z_{-\boldsymbol{m}}^{I} Z_{\boldsymbol{m}}^{I}\right), \\
H_{1}= & \frac{4 \pi i}{\sqrt{N}\left(\alpha^{\prime}\right)^{3 / 2}} \sum_{\boldsymbol{m}, \boldsymbol{n}, \boldsymbol{l}} N \sin \frac{\pi}{N}(\boldsymbol{n} \times \boldsymbol{l})\left(\left(R_{1} m_{2} A_{\boldsymbol{m}}^{2}-R_{2} m_{1} A_{\boldsymbol{m}}^{1}\right) A_{\boldsymbol{n}}^{1} A_{\boldsymbol{l}}^{2}\right. \\
& \left.+Z_{\boldsymbol{m}}^{I}\left(R_{1} m_{2} A_{\boldsymbol{n}}^{1}+R_{2} m_{1} A_{\boldsymbol{n}}^{2}\right) Z_{\boldsymbol{l}}^{I}\right) \delta_{\boldsymbol{m}+\boldsymbol{n}+\boldsymbol{l}_{0},} \\
H_{2}= & \frac{1}{\alpha^{\prime}} \sum_{\boldsymbol{m}, \boldsymbol{n}, \boldsymbol{l}, \boldsymbol{k}} N \sin \frac{\pi}{N}(\boldsymbol{m} \times \boldsymbol{n}) \sin \frac{\pi}{N}(\boldsymbol{l} \times \boldsymbol{k})\left(Z_{\boldsymbol{m}}^{I} Z_{\boldsymbol{n}}^{J} Z_{\boldsymbol{l}}^{I} Z_{\boldsymbol{k}}^{J}\right. \\
& \left.+2 A_{\boldsymbol{m}}^{1} A_{\boldsymbol{n}}^{2} A_{\boldsymbol{l}}^{1} A_{\boldsymbol{k}}^{2}+2 A_{\boldsymbol{m}}^{r} Z_{\boldsymbol{n}}^{I} A_{\boldsymbol{l}}^{r} Z_{\boldsymbol{k}}^{I}\right) \delta_{\boldsymbol{m}+\boldsymbol{n}_{+} \boldsymbol{l}_{+} \boldsymbol{k}, 0_{0}}
\end{aligned}
$$

with $\omega_{\boldsymbol{m}}=\sqrt{\left(R_{1} m_{2}\right)^{2}+\left(R_{2} m_{1}\right)^{2}}$ and $\boldsymbol{m} \times \boldsymbol{n}=m_{1} n_{2}-m_{2} n_{1}$. In the large $\mathrm{N}$ expansion we observe that the leading and the next leading parts $H_{0}$ show the free quadratic behavior. Specially the quadratic terms with respect to $\left(Z_{\boldsymbol{m}}^{I}, \Pi_{\boldsymbol{m}}^{I}\right)$ indicate the Hamiltonian of an infinite set of harmonic oscillators characterized by $\left(m_{1}, m_{2}\right)$. The similar structure was observed in the zero limit of membrane tension in the light-cone Hamiltonian of the supermembrane compactified on $T^{2}$ [11]. The higher order parts $H_{1}$ and $H_{2}$ represent the cubic and quartic complicated interactions. In the large $\mathrm{N}$ limit the energy for the static background is given by $H=P_{+}=2 R N\left(\pi R_{1} R_{2}\right)^{2} / \alpha^{\prime 3}$, where we use the lightcone conventions. This value is also provided from (8) because $\operatorname{Tr}$ can be identified with $N /(2 \pi)^{2} \int_{0}^{2 \pi} d q \int_{0}^{2 \pi} d p$ in the large $\mathrm{N}$ limit, where the trace of quantum operators becomes the phase-space integration of the classical functions. This identification is confirmed by comparing $1 /(2 \pi)^{2} \int d q d p e^{i \boldsymbol{m} \cdot \boldsymbol{p}} e^{i \boldsymbol{n} \cdot \boldsymbol{p}}=\delta_{\boldsymbol{m}+\boldsymbol{n}, 0}, \boldsymbol{p}=(p, q)$ with (10) [1], 12]. Since $P_{+}=M^{2} / 2 P_{-}$with the momentum $P_{-}$quantized to be $N / R$, the membrane mass is given by $M=2 \pi R_{1} R_{2} N /\left(\alpha^{\prime}\right)^{3 / 2}$. Due to the area of membrane $(2 \pi)^{2} R_{1} R_{2} N$ the membrane tension is evaluated as $T_{2}=1 / 2 \pi\left(\alpha^{\prime}\right)^{3 / 2}$ [1, 2].

Now let us return to the Lagrangian $L$ in (7). In the action $S=\int d t L$ the factor $R$ can be absorbed by the definition of the world volume time $t \rightarrow \sqrt{\alpha^{\prime}} t / 2 \pi R$ as well as the rescaling $A_{t} \rightarrow 2 \pi R A_{t} / \sqrt{\alpha^{\prime}}$. Simultaneously we arrive at a Lorentz and gauge invariant form of the action

$$
S=\int d t \operatorname{Tr}\left(\frac{(2 \pi)^{3}\left(\alpha^{\prime}\right)^{3 / 2}}{4} F_{\mu \nu}^{2}-\frac{\pi\left(R_{1} R_{2}\right)^{2}}{\left(\alpha^{\prime}\right)^{5 / 2}}+\frac{\pi}{\sqrt{\alpha^{\prime}}}\left(D_{\mu} Z_{I}\right)^{2}+\frac{\left[Z_{I}, Z_{J}\right]^{2}}{8 \pi\left(\alpha^{\prime}\right)^{5 / 2}}\right) .
$$


Here the large $\mathrm{N}$ limit is taken so that the matrices described by the quantum phasespace variables approach the ordinary functions that depend on the classical phase-space ones. Then the trace identified with $N /(2 \pi)^{2} \int d q d p$ is further rewritten in terms of dual valuables $\left(x_{1}, x_{2}\right)$ as

$$
\frac{1}{(2 \pi)^{2} \hat{R}_{1} \hat{R}_{2}} \int_{0}^{\sqrt{N} \hat{L_{1}}} d x_{1} \int_{0}^{\sqrt{N} \hat{L_{2}}} d x_{2}
$$

where $\hat{R}_{r}=\alpha^{\prime} / R_{r}$ are radii of circles of the dual torus and $\hat{L}_{r}=2 \pi \hat{R}_{r}$. In the large $\mathrm{N}$ limit the effective theory becomes a $\mathrm{U}(1)$ gauge theory in the three-dimensional dual spacetime with a field strength $F_{\mu \nu}=\partial_{\mu} A_{\nu}-\partial_{\nu} A_{\mu}+2 \pi \hat{R}_{1} \hat{R}_{2}\left\{A_{\mu}, A_{\nu}\right\}$ with $\left\{A_{\mu}, A_{\nu}\right\}=$ $\epsilon^{r s}\left(\partial A_{\mu} / \partial x_{r}\right)\left(\partial A_{\nu} / \partial x_{s}\right)$, which seems to include a finite correction term. Similarly $D_{\mu} Z_{I}, \quad\left[Z_{I}, Z_{J}\right]$ are expressed as $\partial_{\mu} Z_{I}+2 \pi \hat{R}_{1} \hat{R}_{2}\left\{A_{\mu}, Z_{I}\right\}, 2 \pi i \hat{R}_{1} \hat{R}_{2}\left\{Z_{I}, Z_{J}\right\}$. The U(1) gauge coupling constant can be read off from (12) and (13) as

$$
g^{2}=\frac{\sqrt{\alpha^{\prime}}}{2 \pi R_{1} R_{2}}
$$

and then has mass dimension $1 / 2$. Moreover the rescaling of integration variables as $t \rightarrow \sqrt{N} t, x_{r} \rightarrow \sqrt{N} x_{r}$ together with $Z_{I}=\left(2 \pi \hat{R}_{1} \hat{R}_{2} \sqrt{\alpha^{\prime}}\right)^{1 / 2} \Phi_{I}$ makes the action (12) except for the second term into the canonical form

$$
S=\sqrt{N} \int d t \int_{0}^{\hat{L_{1}}} d x_{1} \int_{0}^{\hat{L_{2}}} d x_{2}\left(\frac{1}{4 g^{2}} F_{\mu \nu}^{2}+\frac{1}{2}\left(D_{\mu} \Phi_{I}\right)^{2}+\frac{g^{2}}{4}\left[\Phi_{I}, \Phi_{J}\right]^{2}\right)
$$

where $F_{\mu \nu}=\partial_{\mu} A_{\nu}-\partial_{\nu} A_{\mu}+2 \pi \hat{R}_{1} \hat{R}_{2} / \sqrt{N}\left\{A_{\mu}, A_{\nu}\right\}, D_{\mu} \Phi_{I}=\partial_{\mu} \Phi_{I}+2 \pi \hat{R}_{1} \hat{R}_{2} / \sqrt{N}\left\{A_{\mu}, \Phi_{I}\right\}$ and $\left[\Phi_{I}, \Phi_{J}\right]=2 \pi i \hat{R}_{1} \hat{R}_{2} / \sqrt{N}\left\{\Phi_{I}, \Phi_{J}\right\}$. It is seen that the correction term in the $\mathrm{U}(1)$ field strength is suppressed in the large $\mathrm{N}$ limit.

If we take the large $\mathrm{N}$ limit for the Hamiltonian (8) before taking the quantum trace and perform the integration over the classical phase space $(p, q)$, that is, over the two coordinates parametrizing a membrane, then we again have the Hamiltonian identical to (11) with $\sin \frac{\pi}{N}(\boldsymbol{m} \times \boldsymbol{n})$ replaced by $\frac{\pi}{N}(\boldsymbol{m} \times \boldsymbol{n})$. Therefore in comparing two expressions (11) and (15) we interpret (11) as the Hamiltonian describing the membrane wrapped around the rectangular target-space torus, which means that in the separation (11) $U_{1}, U_{2}$ are regarded as winding modes and $A_{1}, A_{2}$ as oscillating modes of the membrane. In this sense in the $(p, q)$ space we recognize that it is better to represent $2 \pi \alpha^{\prime}\left(A_{1}, A_{2}\right)$ by $\left(Z_{1}, Z_{2}\right)$. Recently in Ref. [13] the supermembrane with winding has been studied in the light-cone gauge. The mode decomposition of the Hamiltonian expressed in terms of the structure constants of the group of area-preserving diffeomorphism is in a similar form to our decomposition (11). On the other hand the gauge invariant action (15) shows a system in the dual $\left(x_{1}, x_{2}\right)$ space and $A_{r}$ can be regarded as gauge potentials literally.

The background solution (3) represents a single infinite membrane and yields the $\mathrm{U}(1)$ gauge theory. Now we will consider a background configuration of $N_{2}$ parallel infinite 
membranes which is described by block-diagonal $N \times N$ matrices with $N=N_{1} N_{2}$

$$
U_{1}=\left(-\sqrt{N_{1}} R_{1} p\right) \otimes 1, \quad U_{2}=\sqrt{N_{1}} R_{2} q \otimes 1,
$$

and all others $U_{I}=0, I=3, \cdots, 9$, where $(p, q)$ are $N_{1} \times N_{1}$ matrices with $[q, p]=2 \pi i / N_{1}$ and 1 is the unit $N_{2} \times N_{2}$ matrix. Non-block-diagonal fluctuations are expressed as

$$
\begin{aligned}
Y_{a b}^{1} & =-\sqrt{N_{1}} R_{1} p \delta_{a b}+2 \pi \alpha^{\prime} A_{1}(p, q)_{a b} \\
Y_{a b}^{2} & =\sqrt{N_{1}} R_{2} q \delta_{a b}+2 \pi \alpha^{\prime} A_{2}(p, q)_{a b}
\end{aligned}
$$

with $a, b=1, \cdots, N_{2}$. In the same way as (9) they are expanded as $A_{r}(p, q)_{a b}=$ $1 / 2 \pi \sqrt{\alpha^{\prime}} \sum_{\boldsymbol{m}} A_{a b}^{r}(\boldsymbol{m}) J_{\boldsymbol{m}}$. The commutator between $A_{1}$ and $A_{2}$ is calculated in the large $N_{1}$ expansion [9] as

$$
\begin{aligned}
\left(A_{1} * A_{2}-A_{2} * A_{1}\right)_{a b}= & {\left[A_{1}, A_{2}\right]_{a b}+\sum_{k=1}^{\infty}\left(\frac{\pi i}{N_{1}}\right)^{k} \frac{1}{k !} \sum_{\boldsymbol{m}, \boldsymbol{n}}(\boldsymbol{m} \times \boldsymbol{n})^{k} } \\
& \times\left(A^{1}(\boldsymbol{m}) A^{2}(\boldsymbol{n})-(-1)^{k} A^{2}(\boldsymbol{n}) A^{1}(\boldsymbol{m})\right)_{a b} J_{\boldsymbol{m}+\boldsymbol{n} .}
\end{aligned}
$$

Using the dual coordinates in the leading order of the large $N_{1}$ expansion we have $\left[Y_{1}, Y_{2}\right]_{a b}=2 \pi i R_{1} R_{2} \delta_{a b}+\left(2 \pi \alpha^{\prime}\right)^{2} i\left(F_{12}\right)_{a b}$ where $F_{12}$ is the usual $\mathrm{U}\left(N_{2}\right)$ gauge field strength, and also $D_{t} Y^{r}=2 \pi \alpha^{\prime} F_{t r},\left[Y^{r}, Y^{I}\right]=2 \pi \alpha^{\prime} i D_{r} Z_{I}$ with the standard $\mathrm{U}\left(N_{2}\right)$ covariant derivative. In the large $N_{1}$ limit the $\operatorname{Tr}$ turns out to be $N_{1} /(2 \pi)^{2} \int d p d q t r$ where $\operatorname{tr}$ is the trace for $N_{2} \times N_{2}$ matrices, so that we get a three-dimensional $\mathrm{U}\left(N_{2}\right)$ gauge theory. The suitable rescalings discussed above yield the $\mathrm{U}\left(N_{2}\right)$ gauge action

$$
S=\sqrt{N_{1}} \int d t \int_{0}^{\hat{L_{1}}} d x_{1} \int_{0}^{\hat{L_{2}}} d x_{2} \operatorname{tr}\left(\frac{1}{4 g^{2}} F_{\mu \nu}^{2}+\frac{1}{2}\left(D_{\mu} \Phi_{I}\right)^{2}+\frac{g^{2}}{4}\left[\Phi_{I}, \Phi_{J}\right]^{2}\right),
$$

where $\left[\Phi_{I}, \Phi_{J}\right]$ is the usual $\mathrm{U}\left(N_{2}\right)$ commutator and the $\mathrm{U}\left(N_{2}\right)$ gauge coupling constant also scales as (14). The last term has a favorite coefficient, since the further rescaling $\Phi_{I} \rightarrow \Phi_{I} / g$ leads to $\operatorname{tr}\left(F_{\mu \nu}^{2} / 2+\left(D_{\mu} \Phi_{I}\right)^{2}+\left[\Phi_{I}, \Phi_{J}\right]^{2} / 2\right) / 2 g^{2}$ which is compared with the starting Lagrangian (1).

Let us next consider a four-brane solution expressed in a direct tensor-product form

$$
\begin{array}{ll}
U_{1}=-\sqrt{n_{1}} R_{1} p_{1} \otimes 1, & U_{2}=\sqrt{n_{1}} R_{2} q_{1} \otimes 1, \\
U_{3}=-1 \otimes \sqrt{n_{2}} R_{3} p_{2}, & U_{4}=1 \otimes \sqrt{n_{2}} R_{4} q_{2},
\end{array}
$$

and all others $U_{I}=0, I=5, \cdots, 9$, where $N=n_{1} n_{2}$ and $p_{i}, q_{i}$ are $n_{i} \times n_{i}$ matrices obeying $\left[q_{i}, p_{i}\right]=2 \pi i / n_{i}, i=1,2$. Defining the dual variables as

$$
x_{1}=\frac{\alpha^{\prime} \sqrt{n_{1}}}{R_{1}} q_{1}, x_{2}=\frac{\alpha^{\prime} \sqrt{n_{1}}}{R_{2}} p_{1}, x_{3}=\frac{\alpha^{\prime} \sqrt{n_{2}}}{R_{3}} q_{2}, x_{4}=\frac{\alpha^{\prime} \sqrt{n_{2}}}{R_{4}} p_{2}
$$


and substituting the fluctuations around the four-brane background $Y^{r}=U_{r}+2 \pi \alpha^{\prime} A_{r}, r=$ $1, \cdots, 4$ into the starting Lagrangian (2) we obtain also the expressions (17) and (8) with $r=1, \cdots, 4$ and $I=5, \cdots, 9$ where $F_{12}^{2}$ and $\left(R_{1} R_{2}\right)^{2}$ are replaced by $\sum_{r<s} F_{r s}^{2}$ and $\left(R_{1} R_{2}\right)^{2}+\left(R_{3} R_{4}\right)^{2}$ respectively. The fluctuations $A_{r}$ are also expanded as

$$
A_{r}=\frac{1}{2 \pi \sqrt{\alpha^{\prime}}} \sum_{\boldsymbol{m}, \boldsymbol{n}} A_{\boldsymbol{m}, \boldsymbol{n}}^{r} J_{\boldsymbol{m}}\left(p_{1}, q_{1}\right) \otimes J_{\boldsymbol{n}}\left(p_{2}, q_{2}\right)
$$

The comparison of the trace of quantum operators

$$
\operatorname{Tr}\left(J_{\boldsymbol{m}}\left(p_{1}, q_{1}\right) \otimes J_{\boldsymbol{n}}\left(p_{2}, q_{2}\right) J_{\boldsymbol{m}^{\prime}}\left(p_{1}, q_{1}\right) \otimes J_{\boldsymbol{n}^{\prime}}\left(p_{2}, q_{2}\right)\right)=N \delta_{\boldsymbol{m}+\boldsymbol{m}^{\prime}, 0} \delta_{\boldsymbol{n}+\boldsymbol{n}^{\prime}, 0}
$$

with the four-dimensional phase-space integration

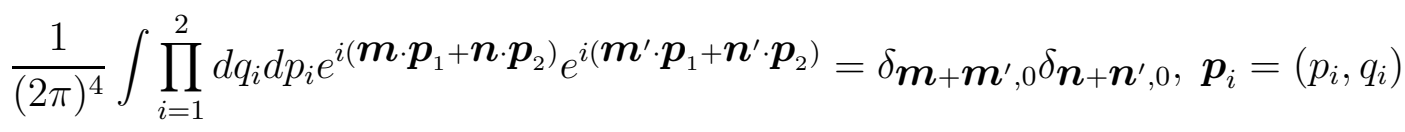

makes $\operatorname{Tr}$ identified with $N /(2 \pi)^{4} \int \prod_{i} d q_{i} d p_{i}$ in the large $\mathrm{N}$ limit. The mass square of the static four-brane configuration is obtained by $M^{2}=4 \pi^{2} N^{2}\left(\left(R_{1} R_{2}\right)^{2}+\left(R_{3} R_{4}\right)^{2}\right) / \alpha^{\prime 3}$. Since the area of the infinite four-brane is given by $n_{1} n_{2} \prod_{r} 2 \pi R_{r}$ the four-brane tension is estimated as $T_{4}=\left(\left(R_{1} R_{2}\right)^{2}+\left(R_{3} R_{4}\right)^{2}\right)^{1 / 2} /(2 \pi)^{3}\left(\alpha^{\prime}\right)^{3 / 2} R_{1} R_{2} R_{3} R_{4}$. If we choose $R_{1} R_{2}=$ $R_{3} R_{4}$ to be $\sqrt{2} \alpha^{\prime}, T_{4}$ becomes $1 /(2 \pi)^{3}\left(\alpha^{\prime}\right)^{5 / 2}$ which combines with $T_{2}=1 / 2 \pi\left(\alpha^{\prime}\right)^{3 / 2}$ to satisfy a relation $T_{p}=\left(4 \pi^{2} \alpha^{\prime}\right)^{3-p} T_{6-p}$ [14, 15, 16] for the $p$-brane tensions $T_{p}$. Through the rescaling $t \rightarrow \sqrt{\alpha^{\prime}} t / 2 \pi R$ a Lorentz invariant action is again obtained to be in the same form as (12) up to the second term. Then in the large $\mathrm{N}$ limit the $\operatorname{Tr}$ becomes

$$
N \int \prod_{i=1}^{2} \frac{d q_{i}}{2 \pi} \frac{d p_{i}}{2 \pi}=\frac{1}{(2 \pi)^{4} \hat{R}_{1} \hat{R}_{2} \hat{R}_{3} \hat{R}_{4}} \int_{0}^{\sqrt{n_{1}} \hat{L_{1}}} d x_{1} \int_{0}^{\sqrt{n_{1}} \hat{L_{2}}} d x_{2} \int_{0}^{\sqrt{n_{2}} \hat{L_{3}}} d x_{3} \int_{0}^{\sqrt{n_{2}} \hat{L_{4}}} d x_{4},
$$

where the size of the four cycles of the four-torus are $L_{r}=2 \pi \hat{R}_{r}$ with $\hat{R}_{r}=\alpha^{\prime} / R_{r}$. In this way the four-brane solution contained in the starting zero-brane theory leads to the five-dimensional $\mathrm{U}(1)$ gauge theory whose coupling constant scales as

$$
g^{2}=\frac{2 \pi\left(\alpha^{\prime}\right)^{5 / 2}}{R_{1} R_{2} R_{3} R_{4}}
$$

with mass dimension $-1 / 2$. The choice $n_{1}=n_{2}=\sqrt{N}$ and the rescalings of $Z_{I}=$ $\left((2 \pi)^{3} \hat{R}_{1} \hat{R}_{2} \hat{R}_{3} \hat{R}_{4} \sqrt{\alpha^{\prime}}\right)^{1 / 2} \Phi_{I}$ as well as the integration variables also provide a compact expression of the canonical gauge action similar to (15) with the five-dimensional integrations multiplied by $N^{3 / 4}$. It is interesting that the behaviors of $g^{2}$ inversely proportional to the volume of the torus in (26) and (14) are similar to the results of Susskind et al. 17, 18, 19 which are obtained by equating the energies stored in the U(1) electric field or magnetic flux in the SYM gauge theory in the dual torus with the corresponding ones in 
the toroidally compactified M(atrix) theory. It is, however, unclear in our framework how a new coordinate emerges for the M(atrix) theory compactified on a two-torus when the torus shrinks to zero size, which particular limit is considered to yield the ten-dimensional type IIB string theory [18, 19].

In conclusion from the D0-brane theory we have derived a sytem that is interpreted in terms of the canonical phase-space variables as the $\mathrm{M}$ (atrix) theory compactified on a two- or four-torus and alternatively represented in terms of the dual phase-space variables as the three- or five-dimensional gauge theory. In the former point of view the four-brane tension with suitable dimension has been presented. We compare our prescription with that of Ganor et al. [5, 20], who replace by hand the matrices $X^{\mu}$ on the $p$-dimensional torus with the covariant derivatives $\nabla^{\mu}=i \frac{\partial}{\partial \hat{x}_{\mu}}+A^{\mu}(\hat{x})$ where $\hat{x}_{\mu}$ are added as the dual extra coordinates on the dual torus. This replacement changes the Lagrangian of the $\mathrm{U}(\mathrm{N})$ $0+1$ dimensional gauge theory into that of the $\mathrm{U}(\mathrm{N}) p+1$ dimensional gauge theory where the dual space integration is added by hand. In their transformed Lagrangian there is no term corresponding to the second term in (12) of our Lorentz invariant Lagrangian.

Recently in Ref. 21] starting from the M(atrix) theory the effective gauge theories on the $p$-brane world volumes have been derived without using T-duality by replacement of covariant derivatives which are interpreted to be expressed in terms of the coordinates parametrizing the $p$-brane rather than the dual coordinates. In their shuffles of matrix fields the linear terms in the gauge potential remain in the effective Lagrangians.

In our approach we have demonstrated that the suitable extractions of the dual coordinates from the basic canonical phase-space variables parametrizing the membrane and four-brane solutions lead to appearances of the dual space intergrations. We have seen that the dual coordinates together with the D2- and D4-brane solutions emerge from only the degrees of freedom of D0-brane, where the trace in the starting Lagrangian contains the dual space integration in a natural way. In the dual spacetime the appropriate rescalings yield the Lorentz and gauge invariant action in a favorite form with the gauge coupling constant characterized by the volume of the dual torus. We expect that our demonstrations will be useful to get a better understanding of the toroidal compactification in the $\mathrm{M}$ (atrix) theory. 


\section{References}

[1] T. Banks, W. Fischler, S.H. Shenker, L. Susskind, Phys, Rev. D55 (1997) 5112.

[2] O. Aharony and M. Berkooz, Nucl. Phys. B491 (1997) 184.

[3] G. Lifschytz and S.D. Mathur, hep-th/9612087.

[4] G. Lifschytz, hep-th/9612223; hep-th/9703201.

[5] O.J. Ganor, S. Ramgoolam and W. Taylor IV, Nucl. Phys. B492 (1997) 191.

[6] W. Tayor IV, Phys. Lett. B394 (1997) 283.

[7] T. Banks, N. Seiberg and S. Shenker, Nucl. Phys. B490 (1997) 91.

[8] M.R. Douglas, hep-th/9610041.

[9] M. Li, hep-th/9612222.

[10] N. Ishibashi, H. Kawai, Y. Kitazawa and A. Tsuchiya, hep-th/9612115.

[11] J.G. Russo and A.A. Tseytlin, B490 (1997) 121; J.G. Russo, Nucl. Phys. B492 (1997) 205.

[12] N. Kim and S.J. Rey, hep-th/9701139.

[13] B. de Wit, K. Peeters and J.C. Plefka, hep-th/9705225.

[14] S.P. de Alwis, hep-th/9607011.

[15] J. Polchinski, Phys. Rev. Lett. 75 (1995) 4724.

[16] M.B. Green, C.M. Hull and P.K. Townsend, Phys. Lett. B382 (1996) 65.

[17] L. Susskind, hep-th/9611164.

[18] S. Sethi and L. Susskind, hep-th/9702101.

[19] W. Fischler, E. Halyo, A. Rajaraman and L. Susskind, hep-th/9703102.

[20] A.A. Tseytlin, hep-th/9701125.

[21] E. Keski-Vakkuri and P. Kraus, hep-th/9706196. 